\title{
GÉNERO, POLITICA Y MIGRACIÓN EN LA AGENDA GLOBAL. TRANSFORMACIONES RECIENTES EN LA REGIÓN SUDAMERICANA
}

\author{
MARÍA JOSÉ MAGLIANO* \\ EDUARDO E. DOMENECH**
}

\begin{abstract}
RESUMEN: Las migraciones femeninas se ubican en la actualidad entre los temas que mayor interés suscitan en el terreno de los estudios migratorios y de género. En los últimos tiempos asistimos a una mayor visibilidad social y política y a un incremento significativo del número de mujeres en los procesos migratorios, fenómeno conocido como «feminización de las migraciones». Este fenómeno, al transformar los patrones de migración internacional, ha implicado la consideración de la «mujer migrante» por parte de distintos actores políticos vinculados a esta cuestión. Este artículo analiza primero el lugar que adquiere la figura de la «mujer migrante» en la emergente agenda global sobre migraciones impulsada por agencias de Naciones Unidas y la Organización Internacional para las Migraciones. Posteriormente, examina dicha figura en aquellos espacios políticos supranacionales constituidos recientemente alrededor de la temática migratoria en la región sudamericana, deteniéndose en la experiencia argentina para discutir ciertos aspectos de la presencia/ausencia de la dimensión de género en la actual política migratoria nacional. En este trabajo se sugiere que la visibilidad que alcanza la «mujer migrante» como actor social relevante en los discursos, recomendaciones y lineamientos de la agenda global y regional sobre migraciones, no modifica la histórica invisibilidad de los procesos que producen y reproducen las desigualdades de género, y que condicionan las realidades migratorias femeninas.
\end{abstract}

PALABRAS ClAVE: Género, migraciones internacionales, agenda política, América del sur, Argentina.

ABSTRACT: Nowadays, female migration has become one of the major issues in Migratory and Gender Studies. In the last years, the number of women in migratory processes has increased as well as their social and political visibility, phenomenon known as «feminization of migration». In this context, different political actors related to this topic have considered female migration as an important subject. Firstly, this paper analyzes the place of the "female migrant» in the emergent global agenda on migration promoted by United Nations agencies and the International Organization for Migration. Secondly, it examines that social actor in those supranational political spaces recently constituted in the South American region. Particularly, the Argentinean experience makes possible to discuss the presence/absence of the gender dimension in the current national migratory policy. This article suggests that the visibility of the «female migrant» as a relevant social actor in the discourses, recommendations and lineaments of the global and regional agenda on migration, does not modify the historic invisibility of the processes that produce and reproduce gender inequalities, and which affect female migratory realities.

KEYwORDS: Gender, international migration, political agenda, South America, Argentina.

\footnotetext{
* Becaria del Consejo Nacional de Investigaciones Científicas y Técnicas (Conicet). Docente de la Facultad de Filosofía y Humanidades de la Universidad Nacional de Córdoba, Argentina. E-mail: majomagliano@gmail.com

** Docente-Investigador del Centro de Estudios Avanzados de la Universidad Nacional de Córdoba, Argentina. E-mail: eduardo.domenech@gmail.com
} 


\section{INTRODUCCIÓN}

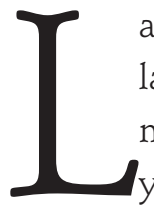

as migraciones femeninas, cuyas especificidades han sido ignoradas en las distintas tradiciones teóricas, se ubican en la actualidad entre los temas que mayor interés suscitan en el terreno de los estudios migratorios

y de género. A pesar que las mujeres siempre han participado en los movimientos poblacionales, históricamente fueron desestimadas como actores sociales relevantes y concebidas como sujetos pasivos. En los últimos tiempos asistimos a una mayor visibilidad social y política y a un incremento significativo del número de mujeres en los procesos migratorios, fenómeno conocido como «feminización de las migraciones». La relevancia de este fenómeno es atribuida no sólo al aumento real de la intervención de la mujer en las migraciones internacionales que ha contribuido a transformar los patrones de migración internacional, sino también a la apertura conceptual que ha hallado la figura de la «mujer migrante» en el ámbito de las ciencias sociales (Oso, 1998: 39). Estos factores han implicado su consideración por parte de distintos actores políticos vinculados a esta cuestión.

Este artículo analiza primero el lugar que adquiere la figura de la «mujer migrante» en la emergente agenda global sobre migraciones impulsada por agencias de Naciones Unidas y la Organización Internacional para las Migraciones (OIM). Posteriormente, examina dicha figura en aquellos espacios políticos supranacionales constituidos recientemente alrededor de la temática migratoria en la región sudamericana, deteniéndose en la experiencia argentina para discutir ciertos aspectos de la presencia/ausencia de la dimensión de género en la actual política migratoria nacional. En este trabajo se sugiere que la visibilidad que alcanza la «mujer migrante» como actor social relevante en los discursos, recomendaciones y lineamientos de la agenda global y regional sobre migraciones, no modifica la histórica invisibilidad de los procesos que producen y reproducen las desigualdades de género, y que condicionan las realidades migratorias femeninas. Los avances de investigación que se presentan en este estudio se apoyan en el examen del discurso de diversas agencias internacionales, principalmente del sistema de Naciones Unidas e instituciones asociadas como la orm, plasmado en documentos de alcance mundial como los informes del Fondo de Población de Naciones Unidas (UNFPA), el Instituto Internacional de Investigaciones y Capacitación para la Promoción de la Mujer (INSTRAW), la Comisión Global sobre Migraciones Internacionales (GCIM) y el Diálogo de Alto Nivel de las Naciones Unidas sobre Migración y Desarrollo; de carácter regional como los informes de la Comisión Económica para América Latina y el Caribe (Cepal) y del Centro Latinoamericano y Caribeño de Demografía (Celade), las conclusiones preliminares del Encuentro Iberoamericano sobre Migración y Desarrollo, el Compromiso de Montevideo de la Cumbre Iberoamericana, y declaraciones finales de la Conferencia Sudamericana sobre Migraciones; y de naturaleza nacional como la normativa migratoria argentina. 
A partir de la década de los setenta, el impulso de los estudios de género en el campo de las ciencias sociales y el desarrollo de movimientos sociales feministas repercutieron en la agenda política mundial. En este contexto, distintos actores locales y globales pusieron en evidencia la invisibilidad de las mujeres en diversos procesos sociales, demandando la implementación de políticas públicas que incorporen la dimensión de género para atender a las problemáticas que enfrentan las mujeres en diferentes contextos sociales. El impacto social y político de estas demandas se reflejó en la primera convocatoria que hacen las Naciones Unidas en 1975 a una Conferencia Mundial sobre la situación de las mujeres (García y Valdivieso, 2005: 42).

Sin embargo, se debieron esperar casi tres décadas para que la figura de la «mujer migrante» adquiriera visibilidad en la agenda global sobre migraciones. ${ }^{1}$ La trascendencia que cobraron los debates feministas, acompañados por las transformaciones en los movimientos internacionales de población, fueron factores que permitieron redefinir a la «mujer migrante» como una protagonista central de los procesos migratorios. En este marco, en los últimos años el género se convirtió en un eje transversal de todas las recomendaciones de las agencias dedicadas a las migraciones internacionales. Esto se visualiza, por ejemplo, en el informe de la UNFPA del año 2006, destinado enteramente a la temática de las migraciones femeninas, o en la multiplicación de investigaciones referidas a las mujeres migrantes en otros organismos de Naciones Unidas, como la INSTRAW. En un documento de trabajo publicado por esta institución en el año 2006 sobre género, remesas y desarrollo, se manifiesta que es fundamental integrar el enfoque de género de manera transversal en los estudios, programas, proyectos y políticas. Desde el ámbito regional, y en consonancia con la agenda mundial, la Cepal ha incluido esta perspectiva en una gran cantidad de informes realizados en los últimos años sobre las migraciones en América Latina y el Caribe (Cepal, 2000, 2003, 2005,

\footnotetext{
${ }^{1}$ Los primeros estudios académicos sobre las migraciones femeninas se desarrollan a partir de la década de los sesenta del siglo xx. Estos trabajos consideraban a la mujer en el contexto de la familia, por lo general ausente de la primera fase del proceso migratorio que aparecía dominado por el hombre. En la década de los ochenta, una investigación sobre migrantes yugoslavas en Alemania, Francia y Suiza cuestionó algunos de estos presupuestos: en primer lugar, reveló que la mujer estaba presente en la primera ola de inmigración y que incluso era, en muchas ocasiones, la iniciadora del proceso; en segundo lugar, determinó que los motivos de la migración no se reducían sólo a vínculos familiares o estrategias económicas de la familia, sino que también podían ser resultado de la propia decisión de la mujer de movilizarse (Morokvasic, 1988, citado por Timur, 2000). A partir del último decenio del siglo pasado, como resultado de una serie de transformaciones globales en las relaciones de producción y reproducción, se manifiesta un interés creciente por la figura de la «mujer migrante». En este sentido, en la actualidad la gran mayoría de las investigaciones que estudian a la mujer en los procesos migratorios coinciden en señalar el carácter transversal de la dimensión de género (Papi, 2003; Parella, 2003), y la necesidad de abordar la problemática de la migración femenina desde una perspectiva multidimensional, que tenga en cuenta el género pero también otras formas de identificación social, como la etnia y la clase social (Parella, 2003; 2005).
} 
2006). La centralidad que ocupa la dimensión de género se observa también en las conclusiones preliminares del Encuentro Iberoamericano sobre Migración y Desarrollo del año 2006, al recomendar que «en futuros encuentros sobre migración se aborde el tema de género con un enfoque transversal, presente en todos los análisis, y no sólo como un asunto específico» (Encuentro Iberoamericano sobre Migración y Desarrollo, 2006: 16).

A partir del reconocimiento del género como un aspecto clave a tener en cuenta en los análisis sobre migraciones internacionales, la agenda política global impulsada por Naciones Unidas demanda la formulación de políticas que atiendan esta dimensión. El informe de la GCIM del año 2005, en esta línea, destaca la necesidad de instrumentar "políticas y programas migratorios que tengan en cuenta el género, que presten atención a la situación social y a la inclusión de las mujeres migrantes y que garanticen que la experiencia migratoria independice a las mujeres» (Capítulo 4, Art. 1). La orm, por su parte, indica que el «género es quizás el factor más importante que moldea las experiencias de los migrantes -más importante que su país de origen y destino, su edad, clase, raza o cultura-. Como tal, afecta muchas políticas y programas que los gobiernos implementan, no sólo en el campo de la migración» (OIM, citado por UnFPA, 2006: 10).

Estas recomendaciones de políticas se inscriben en una perspectiva que asume una nueva manera de organizar, clasificar y controlar los movimientos internacionales de población, basada fundamentalmente en la noción de equilibrio y eficacia: la gestión de las migraciones (migration management). Desde este enfoque, apoyado en buena medida en la lógica costo-beneficio - encarnada en la figura del «balance contable» (Sayad, 1998) - y bajo una visión pragmática que reconoce la imposibilidad de controlar eficazmente (en el sentido de «restricción») los flujos migratorios, especialmente aquellos de carácter «irregular», los migrantes son clasificados en base a una supuesta (in)utilidad, estableciendo una serie de «ventajas» o «beneficios» $\mathrm{y}$ «desventajas» o «perjuicios» (no sólo económicos) de la migración. La novedad es que ahora las migraciones son consideradas mayormente en términos de «oportunidad» (se desplaza aquí la noción de «problema» asociado a las migraciones durante décadas en la agenda mundial), destacando por un lado la "vulnerabilidad de los migrantes» y acentuando por el otro las oportunidades que ofrecen, atribuyéndoles una capacidad de transformación inaudita: el papel de los migrantes en el crecimiento económico, el desarrollo y la reducción de la pobreza. ${ }^{2}$ Consecuentemente, desde esta perspectiva se clasifica a los flujos migratorios

\footnotetext{
2 Bien sabemos que una cuestión es afirmar que la migración internacional contribuye a la economía global y que su expansión depende de la movilidad de mano de obra y otra muy diferente es sostener que el aporte de los migrantes al crecimiento de la economía mundial representa un progreso para el bienestar general, incluidos los sectores y clases sociales que se encuentran bajo condiciones de explotación y exclusión social, entre ellos los «trabajadores migrantes». La expresión "Managing Migration for the Benefit of All» resume bien la posición que sostienen ciertas agencias de Naciones Unidas.
} 
entre «deseables» $\mathrm{y}$ «no deseables» en nombre del «desarrollo» de acuerdo a su carácter ordenado/desordenado, voluntario/forzoso y reducido/masivo. ${ }^{3}$

El tratamiento de la migración femenina, específicamente, no se aparta de estos lineamientos. En general, el discurso de la agenda política mundial promovida por las diversas agencias de Naciones Unidas identifica a las «mujeres migrantes» como "grupo vulnerable» y subraya los aportes o beneficios económicos y sociales de la migración femenina, tanto para las sociedades de origen como las de destino. Junto a ello, señala la necesidad que los flujos migratorios sean «ordenados» $\mathrm{y}$ «legales», a la vez que resalta los efectos negativos que otro tipo de desplazamiento pudiera ocasionar al país de origen, de destino y a la mujer migrante. Estos efectos serían soportados por las propias migrantes, en especial aquellas que se desplazan sin autorización legal y que pueden sufrir una mayor desprotección y explotación en los países de llegada. Según este enfoque, el aprovechamiento de los aspectos considerados positivos de la migración femenina y la atenuación de la «vulnerabilidad» de las mujeres migrantes dependen precisamente de las características de las migraciones: éstas deberían ser «ordenadas» y «encauzadas». Esto evitaría la desprotección, la violencia, la trata de personas y la explotación sexual que padece un alto porcentaje de las mujeres que se movilizan. En esta dirección, el informe de la UNFPA sugiere que «al eliminar las disposiciones discriminatorias y asegurar que las mujeres tengan oportunidades de migrar legalmente es posible contribuir a reducir la migración irregular, reducir el contrabando y la trata de personas y posibilitar que las mujeres apoyen a sus familias sin correr riesgos indebidos» (UNFPA, 2006: 70). Asimismo, expresa que «el reconocimiento explícito de las necesidades humanas de las mujeres y la necesidad de lograr la igualdad entre hombres y mujeres es un requisito básico de cualquier marco normativo racional, equitativo y eficaz que trate de encauzar la migración de manera ordenada y humana» (UNFPA, 2006: 1).

En correspondencia con la perspectiva de la gestión de las migraciones, la migración femenina es percibida como un factor de desarrollo para las comunidades de origen y de destino. Al respecto, desde la orm se establece que

las mujeres migrantes son el elemento principal de la ecuación migración-desarrollo en virtud del uso de sus diversas aptitudes y de las transferencias que realizan a sus países de origen. Para apoyar su participación en el desarrollo es necesario mejorar su posición económica y social tanto en los países receptores como en los países de origen (Ndiaye, 2006: 23).

El documento de la UNFPA, por su lado, manifiesta que «los problemas de la inmigración y del desarrollo van de la mano. El aumento de las inversiones en reducir la pobreza, propiciar la igualdad entre hombres y mujeres y fomentar el desarrollo

${ }^{3}$ Véase Domenech (2007) para una primera aproximación a la emergente agenda global sobre migraciones internacionales. 
(...) son factores necesarios para lograr un sistema más ordenado de migración» (UNFPA, 2006: 3).

Por otra parte, la relación entre migración femenina y desarrollo está influenciada por el impulso de las perspectivas de género en las teorías del desarro1lo. Estos modelos teóricos, que concibieron históricamente a las mujeres como inactivas en la esfera "productiva», se modificaron en los últimos decenios como resultado de cambios en las relaciones de producción y reproducción a nivel internacional (Parella, 2005: 104). El incremento cuantitativo de las mujeres en los flujos migratorios, las tasas de actividad económica de la mujer migrante en la sociedad de destino, los aportes del feminismo alrededor de la posición de la mujer en la sociedad y las relaciones de género, y el envío de remesas de dinero desde los lugares de destino hacia las comunidades de partida, repercutieron en los enfoques sobre el desarrollo, centrando las preocupaciones en revertir la subordinación femenina, a través del empowerment o acceso paulatino de las mujeres al control de los recursos materiales y simbólicos (Parella, 2003: 84, énfasis en el original).

La concepción de la migración femenina como «contribución al desarrollo», está asociada además a la visión que señala que la migración puede ser un factor para muchas mujeres de cambios positivos en las relaciones de género. Esta perspectiva parte del supuesto que la inserción social y laboral en los países de llegada brinda a las mujeres migrantes la posibilidad de transformar los roles y relaciones de género y lograr una mayor autonomía e independencia respecto al lugar de origen. Estas nociones no escapan a la mirada etnocéntrica que considera que la migración desde países periféricos hacia los centrales puede ofrecer a las mujeres mayores posibilidades de modificar sus contextos de subordinación y desigualdad. En este sentido, Gregorio (1998: 192) sostiene que gran parte de los estudios centrados en las migraciones femeninas parten de premisas equivocadas por su etnocentrismo, en la medida en que se da por hecho que la migración significa pasar de una sociedad "tradicional» a una «moderna», lo cual implicaría una mejora para las mujeres. Numerosos estudios han criticado esta tesis puesto que en muchos casos no sólo no se modifica la desigualdad de género, sino que los roles tradicionales se fortalecen (Morokvasic, 1984; Hugo, 2000). No se trata de negar que la migración, en tanto proceso social, puede generar cambios en las relaciones de género pero, como asegura Ariza (2000: 226), la orientación del cambio no debe presuponerse como tampoco su ocurrencia.

Al mismo tiempo que desde distintos organismos internacionales se subrayan los «aportes» de las mujeres migrantes, tanto en las sociedades de partida como en las de llegada, éstas son identificadas como parte de los "grupos vulnerables». Así, el Informe del Diálogo de Alto Nivel de Naciones Unidas sobre Migración y Desarrollo (2006) indica que la migración entraña riesgos que suelen ser mayores para las mujeres que para los hombres y que, por consiguiente, sería importante "adoptar políticas que tuvieran en cuenta las circunstancias y experiencias particulares de las mujeres migrantes y que redujeran su vulnerabilidad a la explota- 
ción y el abuso». Esta concepción, que ubica a las mujeres migrantes como sujetos frágiles y débiles, proclives a la exclusión y marginación social (Agrela, 2006), responde a una visión pasiva de su participación en los movimientos migratorios. La creencia de que no deciden sobre su propia movilidad y que son más propensas a ser «abusadas» $\mathrm{y}$ "explotadas» hace que sean descritas como víctimas hacia las que se despiertan sentimientos de protección y compasión que, según considera Juliano (2002), es otra forma de estigmatización y segregación social. Esta perspectiva que asocia a la «mujer migrante» a los "grupos vulnerables» por el hecho de ser mujer, haciendo hincapié en su pasividad y debilidad a la hora de explicar sus desplazamientos, se apoya en aquellas posturas que naturalizan conductas y roles socialmente establecidos para hombres y mujeres, y no toma en cuenta las distintas estrategias desplegadas por las propias mujeres migrantes para modificar sus trayectorias de vida. ${ }^{4}$

Finalmente, la consideración de la «mujer migrante» por parte de distintos actores sociales y políticos vinculados a las migraciones internacionales no supuso un cuestionamiento del «orden» de género. Los lineamientos y recomendaciones de la agenda global sobre migraciones establecen como prioritaria la necesidad de reconocer la dimensión de género en los programas y políticas migratorias para así modificar la «vulnerabilidad» que afecta a un amplio sector de las mujeres migrantes y potenciar los «aportes» para el «desarrollo» de sus países de origen y destino, pero no discuten el modelo social, económico, político y cultural dominante que ha profundizado, legitimado y reproducido desigualdades de género. Como sostiene Calavita (1989: 175), el debate sobre política migratoria debe ser parte de una discusión más amplia que incluya la política socio-económica en su conjunto.

\section{GÉNERO, MIGRACIÓN Y POLÍTICA EN LA REGIÓN SUDAMERICANA. EL CASO ARGENTINO}

Los procesos migratorios contemporáneos en América Latina están marcados por los efectos de las políticas neoliberales en la región a partir de su aplicación en la década de los setenta y, sobre todo, en los años noventa. El incremento de los niveles de desempleo y los índices de pobreza, la precarización y desregulación laboral, convirtieron a la migración en una estrategia de supervivencia para un importante número de mujeres. El aumento de la participación femenina en los movimientos migratorios de la región, especialmente hacia los países centrales, ha sido un factor clave en la visibilización de la perspectiva de género en la escena política latinoamericana. Este contexto, sumado a la centralidad que ha alcanzado el discurso de la «feminización de las migraciones» a nivel mundial, repercutió

\footnotetext{
${ }^{4}$ Desde los estudios sobre vulnerabilidad social se sugiere que las personas, las familias y los grupos empobrecidos que viven en espacios de exclusión, hacen uso de sus propios recursos en forma continua para mejorar su bienestar y para enfrentar situaciones adversas (Kaztman, 1999: 3).
} 
en la consideración de la «mujer migrante» en la agenda de trabajo regional sobre migraciones. El creciente interés por la "cuestión migratoria» en el ámbito político sudamericano se refleja en la multiplicación de espacios oficiales de encuentro y consulta sobre este tema: la Conferencia Sudamericana de Migraciones y la Cumbre Iberoamericana ilustran este nuevo escenario. ${ }^{5}$

En la Conferencia Sudamericana sobre Migraciones, que comenzó a reunirse en el año 2000 con intervención de la mayor parte de los países de la región, la figura de la «mujer migrante» adquiere cierta visibilidad y especificidad. En la sexta reunión realizada en Asunción, Paraguay, en mayo de 2006, en el marco de las demandas de protección a los derechos humanos de los migrantes, la declaración final establece que se deben «respetar de manera irrestricta los derechos humanos de los migrantes, especialmente los de la mujer migrante y menores no acompañados, con independencia de la situación migratoria» (Art. 1). Además, la migración, conforme a la tendencia internacional de destacar los «beneficios» para el «desarrollo» que pueden ocasionar ciertos flujos migratorios, es percibida como «aporte», tanto para los países de destino como de salida (Art. 3). Por su parte, el Compromiso de Montevideo de la Cumbre Iberoamericana (2006), afirma que «frente al aumento progresivo de la participación de la mujer en las migraciones internacionales, necesitamos adoptar políticas de equidad de género que contemplen el impacto diferenciado que tiene la migración en las mujeres y la necesidad de atender las causas estructurales que la llevan a migrar» (Art. 18). Se compromete también a prestar debida atención a las peculiaridades de la migración femenina, fomentando y garantizando el pleno respeto de los derechos humanos de las mujeres migrantes, en el marco del ordenamiento jurídico de cada Estado en todas las esferas e independientemente de su condición migratoria, así como la eliminación de todas las formas de discriminación y de violencia en su contra (Art. 25 inc. 1). En este sentido, y acorde con la agenda global, persiste en el ámbito regional la consideración de la «mujer migrante» como parte de los «grupos vulnerables», junto con los niños. Esta concepción de «Vulnerabilidad» asociada a las migraciones femeninas manifiesta cómo la propia naturaleza de las diferencias sexuales son dotadas de significado social, naturalizando y reproduciendo estereotipos sociales (Stolcke, 1999).

Sin embargo, es en el ámbito del Estado nacional donde es importante observar las implicaciones de la creciente visibilidad de la figura de la «mujer migrante» puesto que si bien el rol del Estado se ha visto modificado en la arena política internacional, donde la disputa por la producción de sentidos, prácticas y políticas se ha complejizado, mantiene su poder de decisión sobre los asuntos migratorios siendo un actor clave en la formulación y aplicación de la política migratoria. ${ }^{6}$

5 Véase Domenech (2007) para un análisis crítico de la agenda política sudamericana y argentina sobre migraciones.

${ }^{6}$ Aunque los discursos y políticas de ciertas agencias internacionales ejercen una innegable influencia en la construcción de las agendas políticas nacionales también hay que considerar que la 
En particular, el examen del caso argentino resulta de interés en la medida en que la actual legislación migratoria, sancionada en el año 2003 bajo la presidencia de Néstor Kirchner y aún no reglamentada, se concibe como «modelo» de política migratoria en la región.

A pesar que a partir de la instrumentación de esta nueva normativa el posicionamiento del Estado argentino frente a las migraciones internacionales se modifica al adoptar la perspectiva de los derechos humanos, no logra superar la invisibilidad de género que caracterizó a las políticas migratorias implementadas en el país a lo largo del siglo xx. Más allá que esta ley dispone sobre la trata de personas, en consonancia con la agenda internacional sobre migraciones que considera a esta cuestión una de las problemáticas centrales que afrontan las mujeres en los procesos migratorios, no sólo no hace ninguna referencia a la «mujer migrante» ni a ninguna identidad de género, sino que tampoco advierte las formas diferenciadas de inserción y acceso al mercado de trabajo por parte de los y las migrantes. ${ }^{7}$

En primer lugar, si bien la ruptura más evidente que supuso la sanción de esta norma lo constituye la introducción de la perspectiva de los derechos humanos y el consecuente abandono de la doctrina de seguridad nacional vigente desde la última dictadura militar, una de sus mayores paradojas es justamente que, aunque retoma la perspectiva de los derechos humanos, ignora la dimensión de género. ${ }^{8}$ Esto implica la adopción de la concepción tradicional de derechos humanos de Naciones Unidas la cual ha sido discutida desde los enfoques de género por su androcentrismo, en tanto al mantener una supuesta neutralidad de género no hace más que reforzar los procesos de exclusión, discriminación y desigualdad de las mujeres en diversos contextos sociales.

En segundo lugar, esta disposición mantiene una visión acotada sobre el concepto de «trabajo» y sobre quién es considerado «trabajador/a migrante». La actual

agenda global también se construye a partir de casos nacionales que se proponen como «exitosos». Esta agenda no siempre es impuesta de manera unilateral y que las estrategias de negociación de los organismos internacionales pueden resultar sustantivamente diferentes. En el ámbito internacional, a pesar que las iniciativas y medidas estatales pueden responder a recomendaciones y exigencias de organismos internacionales (conformadas a su vez por Estados nacionales), muchas de ellas son producto de compromisos asumidos por el Estado en acuerdos regionales y en reuniones mundiales.

7 La nueva la nueva ley de migraciones define a la trata de personas como un «delito al orden migratorio», estableciendo diferentes penas de acuerdo al tipo de participación que se tenga en la realización y reproducción de esta actividad. En este sentido, denomina "delitos al orden migratorio» a los que realizaran, promovieran o facilitaran el tráfico ilegal de personas, imponiendo penas de uno a seis años para las personas que se dedican a esta actividad (Ley de migraciones, 2003, Art. 116), agravándose las penas de cinco a quince años «cuando se hubiere puesto en peligro la vida, la salud o la integridad de los migrantes o cuando la víctima sea menor de edad; y de ocho a veinte años cuando el tráfico de personas se hubiera efectuado con el objeto de cometer actos de terrorismo, actividades de narcotráfico, lavado de dinero o prostitución» (Ley de Migraciones, 2003, Art. 121).

8 A partir de la presidencia de Néstor Kirchner (2003-2007), la cuestión de los derechos humanos ocupa un lugar central en la agenda política nacional, fundamentalmente en respuesta a las demandas de los organismos de derechos humanos que han reclamado por los actos cometidos durante la última dictadura militar (1976-1983). 
legislación entiende por «migrante» al «extranjero que desee ingresar, transitar, residir o establecerse definitiva, temporaria o transitoriamente en el país conforme a la legislación vigente» (Art. 2). Específicamente, denomina «trabajador migrante» a aquel que «ingrese al país para dedicarse al ejercicio de alguna actividad lícita, remunerada, con autorización para permanecer en el país por un máximo de tres (3) años, prorrogables, con entradas y salidas múltiples, con permiso para trabajar en relación de dependencia» (Art. 23, inc. a). Esta noción de «trabajador migrante», que aparece como neutral a las diferencias de género, deja afuera a un amplio sector de migrantes, en especial a las mujeres que se dedican a ocupaciones no reconocidas por la ley, tanto aquellas que son remuneradas pero sobre las cuales existen pocas normas, como el trabajo sexual; como aquellas que no son remuneradas, como el trabajo «reproductivo» realizado en el espacio doméstico, el cual ha sido históricamente invisibilizado por el Estado como "trabajo» en la medida en que no se recibe un salario por su prestación. ${ }^{9}$ Esto se relaciona con la tradicional construcción social del modelo de «trabajador» que presupone, siguiendo a Pateman (1995), un hombre proveedor económico y protector de la familia que cuenta con una mujer, la esposa, que a cambio atiende sus necesidades reproductivas. Si bien el migrante no sólo es un «trabajador»-aunque muchas veces desde el Estado se lo interpela exclusivamente en esos términos (Sayad, 1998: 55)- la falta de consideración en las políticas migratorias de las implicaciones que entraña la relación género/trabajo puede profundizar la explotación que sufre gran parte de las mujeres migrantes.

En tercer lugar, si bien esta política migratoria establece como uno de sus objetivos fundamentales la regularización de los migrantes, en sintonía con la perspectiva de la gestión de las migraciones, no contempla la informalidad que caracteriza el empleo femenino, siendo esto un limitante de peso para la obtención de la documentación y, en consecuencia, para la regularización migratoria. Precisamente, las mujeres migrantes se concentran -como resultado de la segmentación del mercado laboral en función del género, la etnia y la clase social- en actividades específicas del mercado de trabajo, principalmente en aquellas de carácter informal y de baja calificación. A su vez, las principales tareas desempeñadas por las mujeres migrantes en Argentina como el trabajo doméstico y sexual, la venta ambulante y las labores agrícolas y textiles son ámbitos escasos de legislación y en caso de que exista, generalmente no se cumple. Debido a ello, al distinguirse las actividades femeninas por su precariedad y la falta de contratos laborales, las propuestas que omiten o niegan esta realidad terminan fomentando la irregularidad y la informalidad, confinando a muchas mujeres a la explotación o la exclusión (Juliano, 2006: 211).

\footnotetext{
9 El trabajo reproductivo simboliza el trabajo doméstico y de cuidados que se realiza en el marco del hogar y la familia. La ausencia de un salario, el hecho de ser un trabajo definido socio-culturalmente como femenino y de permanecer invisible incluso a los ojos de las personas que lo llevan a cabo son algunas de sus características principales (Carrasquer et al., 1998: 96).
} 
En Argentina, la «informalización» del mercado de trabajo que acompañó la adopción de las políticas neoliberales a partir de los setenta debe ser vista, siguiendo a Sassen (2003: 178), dentro del fenómeno de la reestructuración económica que ha contribuido a la caída del complejo industrial del periodo de posguerra, pasando de una economía basada en el sector manufacturero a una basada, sobre todo, en el sector de servicios. El proceso de desindustrialización iniciado por la dictadura militar en la década de los setenta produjo importantes cambios en la estructura social argentina a través de la expulsión de mano de obra del sector industrial al sector terciario y cuentapropista, y la constitución de una incipiente mano de obra marginal (Svampa, 2005: 23). Este escenario se agudizó durante los noventa, momento en que la pauta general fue el incremento de la productividad con escasa generación de empleo y deterioro creciente de las condiciones laborales (Svampa, 2005: 34). En este contexto, una gran parte de los migrantes que ingresaron en los últimos decenios a la Argentina -fundamentalmente aquellos procedentes de los países de la región- se han concentrado en el sector informal de la economía, siendo parte de esa "mano de obra marginal», muchas veces en situaciones de irregularidad migratoria.

Desde la sanción de la nueva ley el trámite para obtener la regularización se ha simplificado, en especial para los ciudadanos miembros de los países del Mercosur (Mercado Común del Sur) y sus estados asociados, y con sólo mínimos requisitos se puede adquirir una residencia "precaria» que puede ser renovable y bajo determinadas condiciones puede transformarse en residencia «temporaria» 0 "permanente» (Art. 20). ${ }^{10}$ La posibilidad de obtener la «residencia permanente» queda reducida para los cónyuges e hijos de ciudadanos argentinos (Art. 22) y para aquellos que posean un "medio lícito de vida». Así, el Programa Nacional de Normalización Documentaria Migratoria para extranjeros nativos de los estados parte del Mercosur y sus estados asociados (Disposición N 53253/2005), de diciembre de 2005, dispone que

antes del vencimiento del plazo de la residencia temporaria otorgada a través del presente Programa, los beneficiarios podrán solicitar su conversión a residencia permanente acreditando: carencia de antecedentes penales en la República Argentina; medios lícitos de vida; pago de la tasa correspondiente; declaración jurada de haber permanecido en el territorio nacional durante un lapso mayor al 80\% del tiempo del beneficio otorgado (Art. 17).

En este sentido, la obtención de la «residencia permanente» está sujeta a la posibilidad de demostrar «medios lícitos de vida», que supone la participación en

10 De acuerdo al sistema clasificatorio que establece el Estado, «los extranjeros serán admitidos para ingresar y permanecer en el país en las categorías de «residentes permanentes», «residentes temporarios», o «residentes transitorios»» $\mathrm{y}$ "hasta tanto se formalice el trámite correspondiente, la autoridad de aplicación podrá conceder una autorización de «residencia precaria» (...)» (Ley de Migraciones, 2003, Art. 20). 
el mercado laboral formal, relegando a un número importante de mujeres que intervienen casi exclusivamente en el sector informal de la economía o que llevan a cabo actividades no consideradas por el Estado ni «trabajo» ni «lícitas». La «informalidad» que distingue al trabajo femenino migrante conduciría de esta forma a la «irregularidad» que tanto se pretende combatir con esta legislación.

Por último, esta política migratoria contempla el derecho de reunificación familiar, determinando que «(e)l Estado garantizará el derecho de reunificación familiar de los migrantes con sus cónyuges, hijos solteros menores o hijos mayores con capacidades diferentes» (Art. 10). La importancia del derecho de reunificación familiar y de la «familia» del migrante se planteó en el Encuentro Iberoamericano sobre Migración y Desarrollo, donde la delegación argentina expuso que «(e)l Estado garantizará el derecho de reunificación familiar de los migrantes con sus padres, cónyuges e hijos, entendiendo que la familia es un ámbito de contención necesario e importante para todo migrante» (Una experiencia Argentina para América del Sur. Plan Patria Grande, Encuentro Iberoamericano sobre Migración y Desarrollo, 2006).

Las políticas de reunificación familiar han sido discutidas desde la perspectiva de género puesto que han fomentado históricamente la dependencia de la mujer a la unidad familiar. En esta perspectiva, Gil Araujo (2006) sostiene que para las mujeres que ingresan a otros países por esta vía la legislación, en líneas generales, ha reproducido la tradicional concepción de la dependencia femenina a una esfera privada regulada por un hombre. Esta visión, vinculada a la mujer dependiente que migra en contextos familiares, se basa en las posturas clásicas que explican la presencia de la mujer en los fenómenos migratorios a partir de la dicotomía «público/privado». La separación "público/privado», ejemplificada en la separación espacial e institucional del lugar de trabajo asociado al hombre, y la esfera doméstica asociada a la mujer, afirma Maquiera (2001: 52), se consolida con el desarrollo de la industrialización y con las justificaciones ideológicas que acompañaron a este proceso. ${ }^{11}$ En efecto, estas nuevas condiciones estructurales, cuyas consecuencias sociales fueron legitimadas por las teorías liberales de la época, supusieron por un lado, el confinamiento parcial de muchas mujeres a las actividades de sus hogares y su exclusión del mercado de trabajo, siendo las labores domésticas concebidas como «no trabajo» porque no se intercambian por un salario; por otro, la invisibi-

\footnotetext{
11 Lo «doméstico» y lo "privado» no son sinónimos. Murillo (2006: 20), en este sentido, cuestiona el binomio «público/privado», incluyendo en este «matrimonio» al espacio «doméstico», ya que señala que "privado» no equivale a "doméstico», más aún aquellos o aquellas que disfrutan de algún tiempo privado tienen resuelto (por vía propia o por delegación) la infraestructura doméstica, de lo contrario «su» tiempo estará sujeto a restricciones. El Estado nacional, en su afán de mantenerse «neutral» a lo que sucediera de las puertas de la casa «hacia adentro» se convirtió en un actor clave en la reproducción de la dicotomía "público/privado», invisibilizando el trabajo "doméstico», en tanto era parte del mundo «privado». Esta supuesta neutralidad del Estado respecto a la regulación del espacio «doméstico» fue fuertemente discutido desde los estudios de género. Para profundizar sobre este tema ver: Pateman, 1995; Maquiera, 2001; Sánchez, Beltrán y Álvarez, 2001; Murillo, 2006.
} 
lización del rol «productivo» de las mujeres, como consecuencia de las nociones dominantes que las definieron como económicamente inactivas.

En esta legislación se exige a la persona que solicita la reunificación familiar la presentación de documentos que acrediten «la solvencia económica del llamante». Para esto demanda los «últimos seis recibos de haberes o certificado contable de ingresos de los últimos seis meses legalizado por el Consejo Profesional de Ciencias Económicas y toda otra documentación que acredite sus ingresos», desconociendo que un alto porcentaje de los y las migrantes trabajan en la economía informal, lo que dificulta el cumplimiento de esta exigencia. En este caso, como señala Parella (2003: 208), la no posesión de un contrato de trabajo implicaría, para muchas mujeres, la imposibilidad de acceso a determinados derechos. Por otra parte, podrán hacer uso de este derecho solamente las personas que fuesen cónyuges u otras personas a quienes la legislación local, provincial, nacional e internacional les otorguen derechos asimilables al matrimonio de los residentes permanentes o temporarios que demanden la reunificación (CELS, 2005), descartando otros modelos de familia existentes en la sociedad actual, fortaleciendo de esta manera la concepción clásica de familia nuclear conformada por padres e hijos.

Si bien el Estado se compromete a "garantizar la reunificación familiar» estableciendo que «la familia es un ámbito de contención necesario e importante para todo migrante» (Encuentro Iberoamericano sobre Migración y Desarrollo, 2006), tanto la definición de familia acotada a la familia nuclear como los requisitos económicos que fija, impide que un amplio número de migrantes pueda reclamar el reagrupamiento formalmente.

En suma, la política sobre migración vigente en Argentina continúa sustentada en el concepto de "hombre migrante», ignorando no sólo las trayectorias y dinámicas migratorias femeninas sino también las condiciones sociales específicas de la situación de la «mujer migrante». El hecho de que se la considere como un sujeto vulnerable, dependiente y pasivo y que no se contemple sus formas de inserción social y laboral, dificulta la obtención de la documentación requerida por el propio Estado, fomentando la irregularidad y la informalidad, y también la dependencia familiar, cuando la reunificación familiar resulta la única vía para acceder a la «residencia permanente». Por lo tanto, las políticas de migración, al omitir la dimensión de género, se convierten en un factor más de exclusión de la «mujer migrante» en la medida en que condicionan su incorporación social, política y económica.

\section{REFLEXIONES FINALES}

En los últimos años, la mayor visibilidad que alcanza la figura de la «mujer migrante» en la agenda política global sobre migraciones no estuvo acompañada de una discusión profunda sobre las causas estructurales que han producido y reprodu- 
cido desigualdades de género. De este modo, diversos actores sociales y políticos, nacionales y supranacionales, admiten la relevancia de la "mujer migrante», redefiniéndola como una protagonista central de las migraciones internacionales, pero no cuestionan el orden social, económico, político y cultural hegemónico, reforzando determinadas representaciones sociales acerca de las migraciones femeninas y la «mujer migrante». La visión que predomina, en el marco de la vinculación entre migración y desarrollo, es una concepción «positiva» del fenómeno, siempre y cuando los flujos sean «encauzados», apoyada en los «aportes» que comporta una migración femenina «ordenada» para el «desarrollo» de los países de origen y destino. Pero, a la vez que se destacan las «contribuciones» de la «mujer migrante» en el proceso migratorio, se las identifica como parte de los "grupos vulnerables», reproduciendo aquellas nociones que las definen como actores sociales dependientes, pasivos y débiles.

La actual normativa migratoria en Argentina, si bien significó un cambio importante en la concepción sobre las migraciones que define y defiende el Estado nacional, no se aleja de estas representaciones sobre la figura de la «mujer migrante» $y$, a pesar que incorpora la cuestión de la trata de personas en conformidad con la agenda política promovida por la OIM, desconoce algunas de las principales problemáticas que enfrentan las mujeres migrantes.

La falta de sensibilidad a la perspectiva de género por parte del Estado en sus políticas públicas migratorias no sólo profundiza la explotación y la marginación social de este sector de la población sino que también fomenta la persistencia de estereotipos sociales respecto al rol de las mujeres en las migraciones. Es por esto que el Estado nacional, a través de sus discursos, prácticas y políticas, resulta un actor central en la producción y reproducción de desigualdades de género. En este contexto, la transformación de estas desigualdades, que se manifiestan en formas de socialización diferenciadas para hombres y mujeres y que determinan la división sexual y social del trabajo, implicaría un cambio radical en la estructura social, política, económica y cultural que ha instaurado y perpetuado la exclusión y subordinación femenina en diversos ámbitos de la sociedad.

\section{BIBLIOGRAFÍA}

Agrela, Belén (2006), «De los significados de género e inmigración (re)producidos en las políticas sociales y sus consecuencias para la acción e integración social», Sesión de Trabajo Migraciones y políticas sociales en España, Universidad Pública de Navarra, Pamplona.

ArIzA, Marina (2000), Ya no soy la que dejé atrás... Mujeres migrantes en República Dominicana, México, Instituto de Investigaciones Sociales/Plaza y Valdés.

Calavita, Kitty (1989), «The Immigration Policy Debate: Critical Analysis and Future Options», en Wayne Cornelius y Jorge Bustamante, (coordinadores), Mexican Migra- 
tion to the United States. Origins, Consequences, and Policy Options, San Diego, Center for US-Mexican Studies, University of California.

Carrasouer, Pilar, Teresa Torns, Elisabet Tejero y Alfonso Romero (1998), «El trabajo reproductivo", Papers, núm. 55.

Cels (2005) «La instrumentación y reglamentación de la nueva Ley de Migraciones», La situación de los derechos humanos en Argentina. Informe Anual 2005, Buenos Aires, Siglo XXI Editores.

CEPAL (2006), Migración internacional, derechos humanos y desarrollo, Chile.

Conferencia Sudamericana de Migraciones (2000-2007), Declaraciones finales.

Cortés Castellano, Patricia, (2005), Mujeres migrantes en América Latina y el Caribe: derechos humanos, mitos y duras realidades, Chile, Cepal.

Cumbre Iberoamericana, (2006), Compromiso de Montevideo, Uruguay.

DAEREN, Lieve (2000), Género en la migración laboral internacional en América Latina y el Caribe,

Taller internacional sobre mejores prácticas relativas a trabajadores migrantes y sus familias, Chile, Cepal/orm.

DOMENECH, Eduardo (2007), «La agenda política sobre migraciones en América del sur: el caso de la Argentina», Revue Européenne des Migrations Internacionales, núm. 23.

Encuentro Iberoamericano sobre Migración y Desarrollo (2006), Conclusiones preliminares, Madrid.

Encuentro Iberoamericano sobre Migración y Desarrollo (2006), Una experiencia Argentina para América del Sur. Plan Patria Grande, Exposición de la Delegación de la República Argentina Madrid.

García, Carmen y Magdalena Valdivieso (2005), «Una aproximación al movimiento de mujeres en América Latina. De los grupos de autoconciencia a las redes nacionales y transnacionales», Osal, año 7, núm. 18.

GCIM (2005), Las migraciones en un mundo interdependiente: nuevas orientaciones para actuar, Ginebra.

Gil Araujo, Sandra (2006), "Las políticas de integración de inmigrantes en el contexto español. Entre la normalización y el derecho a la diferencia», en Fernando Vidal Fernández, (coordinador), Exclusión social y estado de bienestar en España, Barcelona, Icaria.

Gregorio, Carmen (1998), Migración femenina. Su impacto en las relaciones de género, Madrid, Nancea.

Hugo, Graeme (2000), «Migration and Women's Empowerment», en Harriet Presser y Gina Sen, Women's Empowerment and Demographic Processes. Moving Beyond Cairo, New York, Oxford University Press.

Informe del Diálogo de Alto Nivel de las Naciones Unidas sobre Migración y Desarrollo (2006), Nueva York, Naciones Unidas.

instraw (2006), Género, remesas y desarrollo. El caso de la migración de Vicente Noble, República Dominicana.

Juliano, Dolores (2002), «La inmigración sospechosa y las mujeres globalizadas», en Carmen Gregorio y Belén Agrela, (coordinadoras), Mujeres de un solo mundo: globalización y multiculturalismo, Granada, Universidad de Granada.

, Dolores (2006), Excluidas y marginales. Una aproximación antropológica, Valencia, Ediciones Cátedra.

Kaztman, Rubén (1999), Activos y estructuras de oportunidades. Estudios sobre las raíces de la vulnerabilidad social en Uruguay, PNUd-Uruguay, Montevideo, Cepal. 
Ley de Migraciones núm. 25.871, (2003), Argentina.

Maquiera, Virginia (2001), «Género, diferencia y desigualdad», en Elena Beltrán y Virginia Maquiera, (coordinadoras), Feminismos. Debates teóricos contemporáneos, Madrid, Alianza Editorial.

Martínez Pizarro, Jorge (2003), El mapa migratorio de América Latina y El Caribe, las Mujeres y el Género, Serie Población y Desarrollo, Chile, Cepal/Celade.

MorokVASIC, Mirjana (1984), «Birds of Passage are also Women», International Migration Review, núm. 18.

Murillo, Soledad (2006), El mito de la vida privada. De la entrega al tiempo propio, Madrid, Siglo XXI Editores.

NDIAYE, Ndioro (2006), Mujeres, migración y desarrollo, México, Organización Internacional para las Migraciones.

Oso, Laura (1998), Las migraciones hacia España de mujeres jefas de hogar, Madrid, Instituto de la Mujer.

PAPI, Natalia (2003), «Clase social, etnia y género: tres enfoques paradigmáticos convergentes», Utopías, núm. 195, vol. 1.

Parella, Sonia (2003), Mujer inmigrante y trabajadora: la triple discriminación, Barcelona, Anthropos.

(2005), «Segregación laboral y 'vulnerabilidad social' de la mujer inmigrante a partir de la interacción entre clase social, género y etnia», en Carlota Solé y Lluís Flaquer, (coordinadores), El uso de las políticas sociales por las mujeres inmigrantes, Madrid, Instituto de la Mujer.

Pateman, Carole (1995), El contrato sexual, Barcelona, Anthropos/Universidad Autónoma Metropolitana, Unidad Iztapalapa.

Programa Nacional de Normalización Documentaria Migratoria para Extranjeros Nativos de los Estados parte del Mercado Común del Sur y sus Estados Asociados (2005), Disposición núm. 53253/2005, Argentina.

Ramírez, Carlota, Mar García Domínguez y Julia Miguez Morais (2005), Cruzando fronteras: remesas, género y desarrollo, INSTRAW, República Dominicana.

SÁnchez, Cristina, Elena Beltrán, y Silvina Álvarez (2001), «Feminismo liberal, radical y socialista», en Elena Beltrán y Virginia Maquiera, (coordinadoras), Feminismos. Debates teóricos contemporáneos, Madrid, Alianza Editorial.

Sassen, Saskia (2003), Los espectros de la globalización, Buenos Aires, Fondo de Cultura Económica.

SAYAD, Abdelmalek (1998), A imigracão ou os paradoxos da alteridade, São Paulo, Editora da Universidade de São Paulo.

Stolcke, Verena (1999), «己̇Es el sexo para el género como la raza para la etnicidad?», Cuadernos para el Debate, núm. 6.

Svampa, Maristella (2005), La sociedad excluyente. La Argentina bajo el signo del neoliberalismo, Buenos Aires, Taurus.

Timur, Serim (2000), «Cambios en las tendencias y problemas fundamentales de la migración internacional: una perspectiva general de los programas de la UNESCO», Revista Internacional de Ciencias Sociales, núm. 165.

UNFPA (2006), Estado de la Población 2006. Hacia la esperanza. Las mujeres y la migración internacional, Nueva York.

68 PRIMER SEMESTRE 2009

MIGRACIÓN Y DESARROLLO 